\title{
ABUNDANCES OF THE ELEMENTS IN THE HALO STARS, INTERACTION BETWEEN OBSERVATION AND THEORY
}

\author{
M.SPITE \\ Observatoire de Paris-Meudon \\ F-92195 Meudon principal Cedex, FRANCE
}

\section{Introduction}

The halo stars are very old stars formed at the beginning of the evolution of the Galaxy. Their main characteristic is that their atmosphere is very metal-poor since at the time of their birth, the matter of the Galaxy had been enriched by only a few number of supernovae. The first very metal deficient stars have been studied in the sixties: Baschek (1959), Aller and Greenstein(1960) Wallerstein et al. (1963). The stellar models used in these first studies were rather crude: the atmosphere of the stars was represented by only one layer with a mean temperature and a mean electronic pressure, the continuum opacity was supposed to be due to $\mathrm{H}^{-}$and included in addition only the contribution by Rayleigh scattering. Later rescaled solar models were used and finally, at the end of the seventies, grids of theoretical models more and more sophisticated became available.

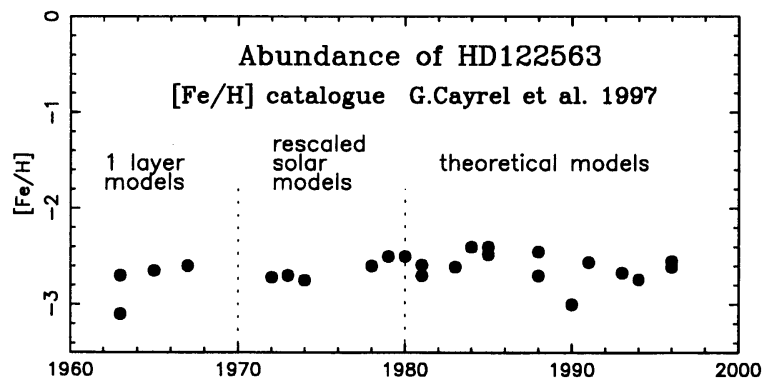

Figure 1: Variation of the metallicity of HD122563 with time

In figure 1 are compared the different determinations of the metallicity of HD122563 versus time. It can be seen that he abundance determinations, as far as an accuracy of $0.2 \mathrm{dex}$ is satisfactory, are robust since even with rather crude models, a good estimation of the metallicity has been obtained. 
In fact, the variation of the iron abundance $[\mathrm{Fe} / \mathrm{H}]$ from author to author is mainly due to the variation of the temperature assumed for the star. When the temperature of the star increases by $250 \mathrm{~K}$ the metallicity of the star increases by about $0.2 \mathrm{dex}$. This effect is in general almost the same for all the elements and thus the ratios like $[\mathrm{Mg} / \mathrm{Fe}],[\mathrm{Ba} / \mathrm{Fe}]$ etc... are more stable.

Let us remark that the important parameter in an abundance determination is not the effective temperature of the star but the temperature in the layers where the metallic lines are formed $(\log \tau \approx-1)$. If two models have the same effective temperature (they predict the same flux) but have different structures the abundances will be different.

Recently difficulties appear with two elements which have in particular an importance for cosmology: lithium and oxygen.

\section{The determination of the lithium abundance.}

The abundance of lithium in the atmosphere of the old halo stars is a test of the Big-bang theory since lithium is essentially built in the primordial nucleosynthesis. The lithium abundance in the halo dwarfs is independent of the metallicity of the star (as far as $[\mathrm{Fe} / \mathrm{H}]<-1.4 \mathrm{dex})$ and independant of the temperature (and thus of the mass) in the interval $5600 \mathrm{~K}<\mathrm{T}_{\text {eff }}<6300 \mathrm{~K}$ (Fig. 2). Two important parameters are: the level of this plateau because if lithium is not depleted in these stars this level represents the Big-bang production, and the spread of the lithium abundance around the plateau since this spread could be the witness of a lithium depletion.

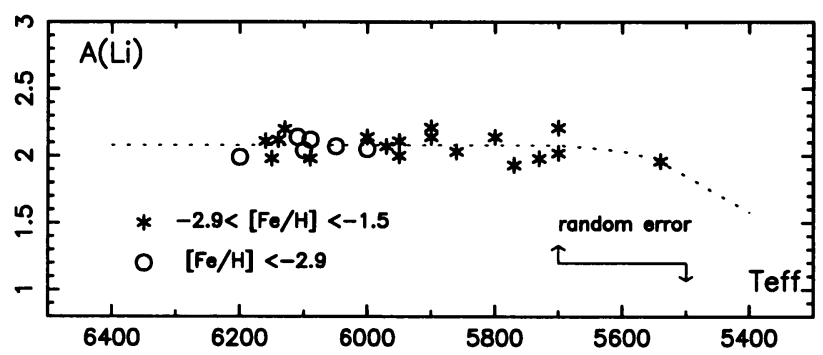

Figure 2: Lithium abundance $\mathrm{A}(\mathrm{Li})$ in halo stars, for $\mathrm{A}(\mathrm{H})=12$.

\subsection{LEVEL OF THE "PLATEAU"}

Since the lithium line is a resonance line of the neutral species of a highly ionised atom, it is very sensitive to the temperature assumed for the star. From author to author, there is a variation of the iron abundance in a given star mainly because they have chosen different effective temperature, on the same way there will be a variation of the lithium abundance. In Spite et 
al. (1996) we have used the new NMARCS models to study a sample of 25 turn-off stars. Three different temperature scales wer used:

-1- The temperature ( Teff $\left._{1}\right)$ was deduced from the excitation equilibrium of the FeI lines and then the level of the lithium plateau was found to be: $\mathrm{A}(\mathrm{Li})=2.08 \mathrm{dex}$ (Fig.2).

-2- The temperature $\left(\mathrm{Teff}_{2}\right)$ was deduced from the (b-y) color and the relation Teff (b-y) computed by Nissen from the NMARCS models, then the level of the plateau was found to be: $\mathrm{A}(\mathrm{Li})=2.15 \mathrm{dex}$.

-3 - The temperature $\left(\mathrm{Teff}_{3}\right)$ was deduced from the profile of the wings of the $\mathrm{H}_{\alpha}$ lines), and we then found that the level of the lithium plateau was: $\mathrm{A}(\mathrm{Li})=2.22$ dex.

(For this sample of stars, mainly turn-off stars, we have found that approximately Teff $_{3}=$ Teff $_{2}+100 \mathrm{~K}=$ Teff $_{1}+200 \mathrm{~K}$ )

These different values of the level of the "plateau" must be compared to the values found by Thornburn 1994: $(\mathrm{A}(\mathrm{Li})=2.3$ using the Carney's scale), by Molaro et al. (1995): $\mathrm{A}(\mathrm{Li})=2.21$, using a temperature deduced from the hydrogen wings, by Ryan et al. $(1996): \mathrm{A}(\mathrm{Li}) \approx 2.18$, using the Carney's scale, and by Bonifacio \& Molaro (1997): $\mathrm{A}(\mathrm{Li})=2.24$ using the IRFM scale.

The problem of the exact level of the plateau of the lithium abundance in the halo stars can be solved only when the temperature scale of the halo stars will be fixed...

\subsection{SPREAD OF THE LITHIUM ABUNDANCE}

Is the spread of the lithium abundance around the plateau explained by the measurement errors, only?

Some sophisticated models predict an almost uniform depletion in the halo stars with $\mathrm{T}_{\text {eff }}>5500 \mathrm{~K}$ (Pinsonneault et al. 1992, Charbonnel \& Vauclair 1995 etc..) but in this case, a dispersion of the lithium abundance around the plateau is expected: (in the rotational models this dispersion would reflect the distribution of the initial stellar rotation velocities).

In our recent analysis we found an rms scatter of $0.08 \mathrm{dex}$, a value expected from the measurements errors only, contrary to the findings of Thornburn (1994) who found in similar conditions a rms scatter of 0.11 0.13 dex.

- The duplicity of some stars can partly explained this discrepancy. Duplicity affects the colors of the star and the equivalent width of the lithium line. In our sample of stars, (Spite et al. 1996) if we only remove the two stars which have been found spectroscopic binaries from our spectra, the rms scatter around the plateau drops by $0.015 \mathrm{dex}$. Since it is estimated that 
TABLE 1. Comparison of the measurements of the equivalent widths of the lithium line

\begin{tabular}{lcccc}
\hline & $\begin{array}{c}\text { Spite et al. } \\
\text { spectra }\end{array}$ & $\begin{array}{c}\text { Thornburn } \\
\text { spectra }\end{array}$ & $\begin{array}{c}\text { Norris et al. } \\
\text { spectra }\end{array}$ & $\begin{array}{c}\text { New meas. of } \\
\text { Norris et al. } \\
\text { spectra }\end{array}$ \\
\hline HD140283 & 48 & 50 & 48 & 48 \\
LTT $815-43$ & 27 & 22 & 15 & 20 \\
CD -33 1173 & 17 & 12 & 10 & 14 \\
\hline
\end{tabular}

more than $20 \%$ of the halo stars are binaries, duplicity may significantly increase the expected spread.

- Moreover, the accuracy of the equivalent widths is often overestimated. The error on the equivalent widths due to photon statistic is given by Cayrel (1988):

$$
\sigma_{W}=\frac{1.5}{S / N} \times \sqrt{F W H M \times \delta x}
$$

where $S / N$ is the signal to noise ratio, $F W H M$ the full width of the line at half maximum, and $\delta x$ the width of one pixel (in $\AA$ ).

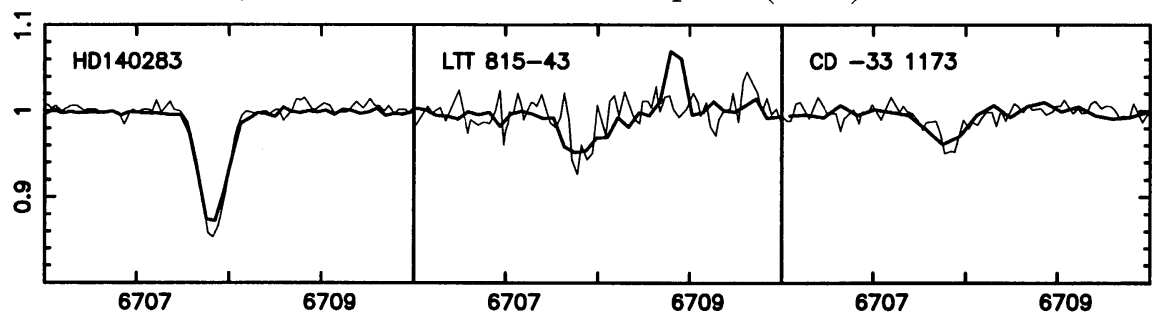

Figure 3: Spectra of three halo stars obtained at ESO by Spite et al.,1996 (thick lines) and by Norris et al. 1994, (thin lines).

In Spite et al. (1996) we observed three stars in common with Norris et al.(1994): HD140283, LTT815-43 and CD-33¹173). The graphs of the observed spectra are given in Norris et al., and it is possible to compare directly the spectra obtained (the resolution is almost the same). The agreement is rather good (Fig. 1). However for the two stars which in the paper of Norris et al. have a lower $\mathrm{S} / \mathrm{N}$ ratio per pixel the difference in the equivalent widths is larger than expected (Table 1).

But, if we repeat the measurements, assuming that the lithium line is a doublet and that the $F W H M$ is the same in the three stars (as it has been observed on our spectra) then the agreement of the equivalent widths is much better. A precise determination of the $F W H M$ is an important 
information when an accurate measurement of the equivalent widths is required. Let us remark that if, in the paper of Thornburn (1994), we select only the stars which have a $\mathrm{S} / \mathrm{N}$ ratio higher than 120 , (and where the $F W H M$ is well defined the observed scatter drops to $\sigma A(L i)=0.083$ a value compatible with the expected errors on the temperature and the equivalent widths.

\section{The determination of the oxygen abundance in the halo stars.}

Oxygen is an abundant element and thus is crucial for the evolution of a star: an oxygen rich star evolves more rapidly than an oxygen poor star with the same metallicity. The oxygen abundance is thus an important parameter to compute the age of the Galaxy.

On the other hand, oxygen is formed in the massive SNII which have a very short life time. On the contrary iron is mainly formed in the SNI which have a much longer life time. The ratio $[\mathrm{O} / \mathrm{Fe}]$ at different ages of the Galaxy gives the ratio SNII/SNI which is a key parameter of the models of the chemical evolution of our Galaxy.

Unfortunately few lines of oxygen can be measured in a spectrum. The forbidden lines at $\lambda=630 \mathrm{~nm}$ (which are almost freee of non LTE effect) are used to determine the oxygen abundance in the giant stars. In the dwarfs they are weak and can be used only if $[\mathrm{Fe} / \mathrm{H}]>-1.5$. In dwarfs with a lower metallicity $-3<[\mathrm{Fe} / \mathrm{H}]<-1.5$ the permitted oxygen lines (a triplet at $\lambda \approx 777 \mathrm{~nm})$ with a very high excitation potential $(\chi=9.14)$, or the $\mathrm{OH}$ molecular bands at $\lambda \approx 360$ or $620 \mathrm{~nm}$ are used.

\subsection{TREND OF OXYGEN ABUNDANCE IN FIELD GALACTIC GIANTS}

Following Gratton \& Ortolani(1986), Barbuy \& Erdelyi-Mendes(1989), Sneden et al.(1991), and Shetrone (1996) the mean value of the ratio [O/Fe] in the field halo giants is $\approx 0.4$ dex (Fig. 4). The decline of $[0 / \mathrm{Fe}]$ toward solar value is interpreted as the growing importance of type I supernovae

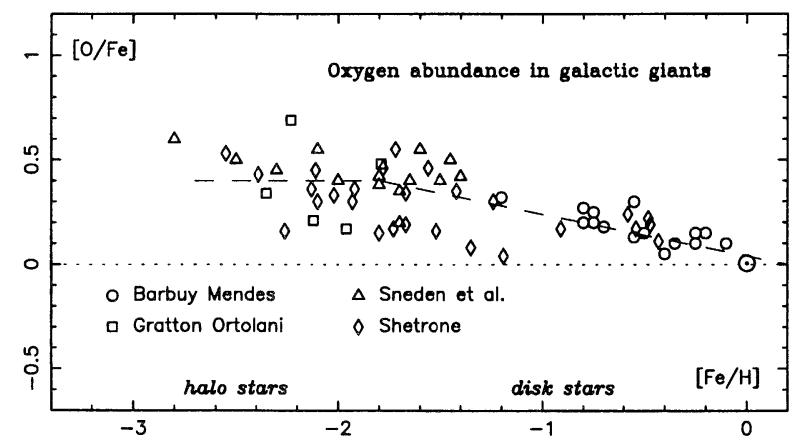

Figure 4: Trend of the oxygen abundance in giant stars 
(where iron is mainly formed) relative to type II supernovae (where oxygen is formed.

\subsection{TREND OF OXYGEN ABUNDANCE IN HALO DWARFS}

Tomkin et al. (1992) and Cavallo et al. (1997) have recently measured the oxygen abundance in halo dwarfs or halo subgiants and found that $[\mathrm{O} / \mathrm{Fe}]$ $\approx 0.8 \mathrm{dex}$, a value significantly higher than the value found in the giant stars. Some years ago Spite \& Spite (1991) and Bessell et al. (1991) have shown that there is indeed a systematic difference between the abundances found from the permitted lines generally used to determine the oxygen abundance in the dwarf stars, and abundances found from the forbidden lines or the $\mathrm{OH}$ molecular bands.

\subsection{WHAT IS THE CAUSE OF THE DISCREPANCY?}

The non-LTE corrections have been calculated by Kiselman (1991) and Tomkin et al.(1992) and are too modest (0.05dex) to explain the observations. Let us remark that the ratio $[\mathrm{O} / \mathrm{Fe}]$ when determined from the permitted oxygen lines is very sensitive to the determination of the temperature of the star. The excitation potential of the oxygen lines $(9.14 \mathrm{eV})$ is higher than the ionisation potential of iron. As a consequence when the temperature of the star is increased, the iron abundance, the oxygen abundance deduced from the forbidden lines or the $\mathrm{OH}$ molecular band, increase but the oxygen abundance deduced from the permitted lines decreases. Moreover the high excitation permitted lines of oxygen are formed deeper in the atmophere than the $\mathrm{FeI}$ lines and thus the ratio $[\mathrm{O} / \mathrm{Fe}]_{\text {permitted }}$ is sensitive to the temperature structure of the model.

It has been also suggested that since the inhomogeneities are larger in the deep layers the neglect of granulation in the computations could explain part of the discrepancy.

Up to now, all the (even recent) papers which have been quoted hereabove, have used old grids of models mainly Bell-Gustafsson (Gustafsson et al. 1975). What about the new grids of models, ATLAS9 or NMARCS ?

\subsection{OXYGEN ABUNDANCES FROM THE NEW GRIDS OF MODELS}

In 1993 King used the ATLAS9 grid of models and showed that shifting the classical colors temperature relations for dwarf stars toward higher temperatures, he could reoncile the oxygen abundance found for dwarfs and giants: $[\mathrm{O} / \mathrm{Fe}]=+0.5$. However there is a difference of about $300 \mathrm{~K}$ between the classical Carney temperature scale and the scale of King. And for many astronomers this large shift appeared to be "ad hoc". 
Recently, Nissen computed from the NMARCS models the relation $\mathrm{T}_{\text {eff }}$ versus $(b-y)$. This relation is close to the relation of Carney, at least for the turn-off stars, and the subgiants. With this relation Nissen et al. (1994) deduced the oxygen abundance from the $\mathrm{OH}$ molecular band in a sample of 9 very metal deficient dwarfs $(-3<[\mathrm{Fe} / \mathrm{H}]<-2)$ and found that in these stars $[0 / F e] \approx 0.58$ dex. But the oxygen permitted lines have been observed for three of these stars by Tomkin et al.(1992) it is thus possible to compute the oxygen abundance from these lines using the same NMARCS model as Nissen et al.(1994). Then, a discrepancy of $\approx 0.3 \mathrm{dex}$ appears between the abundances deduced from the permitted lines and the $\mathrm{OH}$ band.

However in 1996, Nissen (in Spite et al. 1996) taking into account the reddening, gives for the same stars different temperatures. It is possible to estimate the corresponding new value of $[\mathrm{Fe} / \mathrm{H}]$ and of $[\mathrm{O} / \mathrm{Fe}]_{\mathrm{OH}}$ with this new temperature and also to compute the new value of $[\mathrm{O} / \mathrm{Fe}]_{\text {permitted. And }}$ finally now a good agreement is obtained between the permitted lines and the molecular bands: the mean values are $[\mathrm{Fe} / \mathrm{H}]=-2.2,[\mathrm{O} / \mathrm{Fe}]_{\mathrm{OH}}=0.66$ and $[\mathrm{O} / \mathrm{Fe}]_{\text {permitted }}=0.68$. Let us remark however that we could only "estimate" for these three stars the new values of the iron abundance and of the oxygen abundance deduced from the $\mathrm{OH}$ band. It would be worth computing directly these values.

But recently Balachandran and Carney (1996), using ATLAS9 models without overshooting to study HD103095 $([\mathrm{Fe} / \mathrm{H}]=-1.2)$, found also a good agreeement between the $[\mathrm{O} / \mathrm{Fe}]$ ratios computed from the forbidden lines $(+0.33 \mathrm{dex})$ the permitted triplet $(+0.41 \mathrm{dex})$ and the near infra-red $\mathrm{OH}$ and CO lines $(+0.29 \mathrm{dex})$.

When I repeated the computation of the atomic lines with the NMARCS models, the good agreement remained: $\quad[\mathrm{O} / \mathrm{Fe}]_{\text {permitted }}=0.31 \mathrm{dex}$, and $[\mathrm{O} / \mathrm{Fe}]_{\text {forbidden }}=0.36$.

Finally if the values obtained are confirmed, the overabundance of oxygen could be $\approx 0.40 \mathrm{dex}$ at $[\mathrm{Fe} / \mathrm{H}]=-1$ and $\approx 0.65 \mathrm{dex}$ at $[\mathrm{Fe} / \mathrm{H}]=-2$.

\section{Conclusion}

It has been shown that the determinations of critical abundances are reliable only with careful measurements of the equivalent widths, with reliable models, providing a unique determination of $T_{\text {eff }}$, whatever criterium is used (excitation equilbrium, $\mathrm{H}_{\alpha}$ wings, color ...). The improvement of the models (especially for very metal deficient stars) is now an urgent task.

\section{References}

Aller L.H., Greenstein J.L. (1960) ApJS 5, 139

Balachandran S., Carney B.W. (1996) $A J 111,946$ 
Barbuy B., Erdelyi-Mendes M. (1989) $A \& A$ 214, 239

Baschek B. (1959) Zs. f. Ap 48, 95

Bessell M.S., Sutherland R.S., Ruan K. (1991) ApJ 383, L71

Bonifacio P., Molaro P. (1997) MNRAS in press

Cavallo R.M., Pilachowski C.A., Rebolo R. (1997) PASP submitted

Cayrel de Strobel G., Soubiran C., Friel E.B., Ralite N., François (1997) $A G A S$ in press

Cayrel R., (1988) in IAU Symp 132 "The impact of Very High S/N Spectroscopy on

Stellar Physics", G. Cayrel de Strobel and M. Spite eds., Kluwer, p.345

Charbonnel C., Vauclair S. (1995) $A \& A$ 295, 715

Edvardsson B., Anderssen J., Gustafsson B., Lambert D.L., Nissen P.E., Tomkin J. (1993) $A \mathscr{E} A 275,101$

Gustafsson B., Bell R.A., Eriksson K., Nordlund $\AA$ (1975) $A \boxminus A 42,407$

Gratton R., Ortolani S. (1986), $A \& A 169,201$

King J.R. (1993) $A J 106,1206$

Kiselman D. (1991) $A \& A$ 245, L9

Molaro P., Primas F., Bonifacio P. (1995), A $A A$ 295, L47

Nissen P.E., Edvardsson B. (1992) $A \& A$ 261, 255

Nissen P.E., Gustafsson B., Edvardsson B., Gilmore G. (1994) $A \& A 285,440$

Norris J. E., Ryan S. G., Stringfellow G. S. (1994) ApJ 423, 386

Pinsonneault M.H., Deliyannis C.P., Demarque P. (1992) ApJS 78, 179

Ryan S.G., Beers T.C., Deliyannis C., Thorburn J.A. (1996) ApJ 458, 543

Shetrone M.D. (1996) $A J$ 112, 1517

Sneden C., Kraft R.P., Prosser C.F., Langer G.E. (1991) $A J$ 102, 2001

Spite M., Spite F.: (1991) A\&A 252, 689

Spite M., François P., Nissen P.E., Spite F. (1996) $A \& A$ 307, 172

Tomkin J., Lemke M., Lambert D.L., Sneden C. (1992) $A J$ 104, 1568

Thorburn J.A., (1994) ApJ 421, 318

Wallerstein G., Greenstein J.L., Parker R., Helfer H.L., Aller L.H. (1963) ApJ 137, 280

Discussion of this paper appears at the end of these Proceedings. 